\title{
What Are Young Chinese People's Attitudes on Muslims in China and What are the Factors That Influence the Attitude?
}

\author{
Anqi Ma ${ }^{1, *}$, Yufei $\mathrm{Kou}^{2}$, Bingqing $\mathrm{Li}^{3}$, Thong Yang ${ }^{4}$ \\ ${ }^{1}$ Maumee Valley Country Day School, Toledo, 43614, US, 22ama@mvcds.org \\ ${ }^{2}$ Shenzhen Middle School, Shenzhen, 518024, China, 15919499186@163.com \\ ${ }^{3}$ Shanghai Foreign Language School, Shanghai, 200083, China, lbq98856e36@163.com \\ ${ }^{4}$ Changjun High School, Changsha, 410006,China, TYIEONG@163.com
}

\begin{abstract}
This research paper attempts to paint a more detailed picture of Chinese young people's attitudes towards Muslims in China that might help to eliminate stereotypes and improve intergroup relationship. In the study, questionnaire was sent out to 200 Chinese young people to investigate their expressed attitude toward Muslims and Islamic Culture. Overall, the result of the study demonstrates that there is an overall positive attitude toward Muslims in China. In addition, the result also shows generally the participants have a positive stereotypes and a weak feeling of being potentially threaten by Muslims in China. Furthermore, the analysis of different factors including participants' age, sex, ethnical background, and intergroup contact with Muslims reveals that a higher intergroup contact with Muslims in school, neighborhood and other places is highly associated with less negative stereotypes and more positive expressed attitude toward muslims. This result contributes to a deeper understanding of potential effective solutions to eliminate stereotypes faced by Chinese Muslims.
\end{abstract}

Keywords: Muslims, Attitude, Young Chinese People.

\section{INTRODUCTION}

In recent years, with the occurrence of extremist groups that commit acts of violence in the name of Islam, there have been lots of negative perceptions of Muslims and the Islamic faith in many countries. However, despite the fact that there are plenty of studies that contribute to the literature on the global perception of Muslims, such topics are understudied in China. In China specifically, researchers of other studies have found that there is an overall negative framing of news coverage of Muslims and Islam, and non-Muslim Chinese hold a negative stereotype of Muslims and Islam [1]. Thus, the goal of this study is trying to find out specifically what are the young Chinese students' expressed attitudes toward Muslims and Islam in China. Understanding whether people have positive, neutral, or negative attitudes towards Muslims is important as such an understanding is crucial for preventing the negative consequences of intergroup conflicts and discrimination toward Muslims in China [2].

\section{METHOD}

To collect sample, an online anonymous survey was sent to 200 Chinese young people, who are 13 to 22 years old, about their perception of Muslims in China.

In the survey, 29 questions were asked to analyze young Chinese people's expressed attitude toward muslims. To specify the categories of the attitude, the questionnaire is divided into four main parts: potential threat, stereotypes, expressed attitude, and perception of others' attitude. In the dimension of potential threats, the degree of how much the participants believe muslims have potentially threaten their identity and culture is being measured. Within stereotypes, the answers of the participants are investigated to analyze whether they hold a negative, neutral or a positive stereotype toward muslims in China. For the expressed attitude, participants' answers are used to analyze an expressed positive, neutral, or a negative attitude toward muslims in China. Finally, as for perception of other's attitude toward muslims, how respondents perceive others' 
positive, neutral, or negative attitude to ward muslims on social media is being investigated. Besides, there are other background questions in the beginning of the questionnaire which ask about respondents' age, sex, ethnic backgrounds, religious beliefs, and frequency of contact with Muslims in their daily life. The responses of these questions are used for analyzing how different factors may affect people's expressed attitudes toward Muslims. In general, the forms of questions include single choice questions and choosing the statement that suits you. It is worth noting that as the topic of muslims can be sensitive to some of respondents in China, the questionnaire is designed to be anonymous, which is stated at the very beginning of the questionnaire so that the respondents can feel more comfortable expressing their true attitude.

\section{SAMPLE}

Our research group collected 200 pieces of valid feedback from respondents in total. Most of the participants of the sample are aged between 13-22, which is about 75 percent, and only about 25 percent of the participants are 18-22. In addition, there is a relatively lower proportion for male participants $(32 \%)$ than for female participants $(68 \%)$. For ethnic background, most of respondents are identified as Han (83\%) and only about 17 percent of respondents identified as one of the ethnic minority groups in China. Among the ethnic minorities, Hui is the most common ethnic group with a number of 13 respondents, which is around 38 percent of the respondents who identified as one of the 55 ethnic minorities groups in China. For religion, most of the participants $(82 \%)$ do not have religion, 17 percent of the 200 respondents have some religious beliefs, and only one respondent considered him or her as an active member of the person's religious group. In terms of the frequency of contact with Muslims, most of our respondents have a low frequency of contact with Muslims: 59 percent of 200 respondents have no Muslim friends, 56 percent of participants never contact with Muslims in school, 63 percent of the sample never contact with Muslims in the neighborhood, and 59 percent of the sample never contact with Muslims in other places including sports clubs.

\section{PROCEDURE}

In the process of collecting data for the research, an online questionnaire was designed on a Chinese website called WenJuanWang. This online questionnaire allowed the research group to gather up responses from Chinese young people through social medias. Thus, our research group sent the questionnaire through QR code or link on major Chines social medias including WeChat and QQ to our friends, then asking them to send the survey to their friends. By doings so, were created the snowball effect, which allows us to have a big sample of 200 people. The reason that the research group used the Internet's social platform and chose to design the online survey is that they allowed the responses to be gathered more efficiently. After collecting 200 responses from the participants, the research team used both Excel and SPSS to created graphs and to analyze the data.

\section{MEASURE}

\subsection{Analysis of Background information}

At the beginning of the questionnaire, there are eight questions that are set up to indicate respondents' background information, which allow the research group to investigate how different factors may affect people's expressed attitude toward muslims. The first question is about age, and there are two categories: 13 years old to 17 years old, or 18 years old to 22 years old. The second question has two categories: female or male. The question is being asked to investigate potential sex difference in expressed attitude toward muslims. For question three, respondents can indicate their ethnic background by choosing one of the two options: I am a Han person, or I identify as one of the 55 ethnic minority groups. The respondents also filled in blank on the questionnaire to indicate the specific ethnic minority groups that they identify with. The fourth question is about the religious belief of the respondents, and the question has three options that participants can choose: I have no religion, I have some religious belief, and I identify as an active member of a religious group. The fifth question to the eighth question are all designed to investigate the frequency of contact that participants have with muslims, so that the research team can analyze whether there is a statistically difference in attitude toward muslims with respondents of different frequency of contact with muslims - a low frequency of contact or a high frequency of contact. Specifically, the fifth question asked about how many friends the respondents have with four options: no muslims friends, some muslims friends, a lot of muslims friends, or only muslims friends. For the sixth question to the eighth questions, the respondents answer about how often do them have contact with muslim at school, in neighborhood, or in somewhere else. The participants can choose four options for these three questions: never, seldom, some of the time, or most of the time.

The research team used both bar graphs and pie graphs to indicate the distribution of the sample in terms of age, sex, ethnic background, religion, and frequency of contact with muslims.

\subsection{Analysis of Potential Threats}

In the questionnaire, five questions were asked to investigate how much respondents consider muslims as potential threats. The statements are rated on a 5 point 
scale. The response options ranged from strongly agree (1) to Agree (2) to Undecided (3) to Disagree (4) to strongly disagree (5) on a 5-points scale [2]. For question nine, eleven and twelve, a higher scores indicate stronger feelings of symbolic threat, and for question ten and thirteen, lower scores indicate stronger feelings of symbolic threat [2]. In analysis process, the research team did re-code the responses so that a high score of question ten and thirteen is transformed into the corresponding low score on the scale. For example, a score of 4 for question ten and thirteen is transformed into a 2.

\subsection{Analysis of Stereotype}

Participants also answered six questions that indicate their stereotypes toward muslims. To measure stereotypes, research group used six trait adjectives: less educated, dishonest, friendly, socially inferior, generous, and hardworking. Participants were asked to indicate whether they thought these characteristics described Muslims living in China. Each item was rated using a 5point scale ranging from "strongly disagree" (1) to "disagree" (2) to "neutral" (3) to "agree" (4) to "strongly agree" (5). For questions fourteenth, fifteenth, and seventeenth, A higher score indicates more negative stereotypes about Muslims. For questions sixteenth, eighteenth, and nineteenth, a lower score indicates more negative stereotypes about Muslims.

\subsection{Analysis of Expressed Attitude}

To measure respondents' expressed attitude toward muslims in China, the research team designed statements that participants can answer to indicate their expressed attitudes, which can be negative, positive, or neutral. The answers were rated on a 5 point scale. The response options ranged from strongly agree (1) to Agree (2) to Undecided (3) to Disagree (4) to strongly disagree (5) on a 5-points scale. Some sample questions may include "How much do you agree with the statement that the Muslim world has a rich and varied culture" and "How much do you agree with the statement that Discrimination against muslims is unjust." In the analysis, lower scores indicate a more negative attitude toward Islamic culture and Muslims in China. In addition, the last question of the questionnaire was designed as a feeling thermometer that asked respondents to indicate whether they have positive or negative feelings about Muslims living in China. The participants can mark any degree between 0 and 100 . In the analysis, a fifty degrees represents neutral feelings, a score lower than fifty indicates a more negative feelings, and a score higher than fifty indicates a more positive feelings toward muslims.

\subsection{Analysis of Perception of Other's Attitude Toward Muslims}

In the questionnaire, question 27 and 28 are designed to investigate how respondents perceive other's attitude toward muslims in major social media platforms. To be more specific, question 27 asked which one of the four statements that the respondents agreed upon: "There is a negative portrait of Muslims in major social media like Weibo in China(1)" "Do not know (2)" "There is a neutral portrait of Muslims in major social media like Weibo in China(3)" and "There is a positive portrait of Muslims in major social media like Weibo in China (4)." The question is rated on a 4 point scale with lower scores indicating a perception of the more negative attitude of others toward Islamic culture and Muslims in China. For question 28, the participants can choose which one of the four statements that they agreed with: There are lots of negative comments about Muslims on social media. (1) There are some negative comments about Muslims on social media. (2) Do not know. (3) There are some positive comments about Muslims on social media (4) There are lots of positive comments about Muslims on social media (5). In analysis, a five-point scale was used with a lower score indicating a perception of the more negative attitude from others toward Islamic culture and Muslims in China.

\section{RESULT}

\subsection{Descriptive Findings For General Attitude}

For the expressed attitude, the research team first measured mean score for the thermometer, which was 59.23, which is significantly higher than the neutral score of 50. Thus, overall, the participants have a more positive feeling toward muslims. Among the 200 participants, 16 percent of them $(n=33)$ reported scores lower than 50 , indicating a negative feelings towards Muslims, 40 percent of respondents $(n=80)$ reported scores equal to 50 , which represented a neutral feeling, and 44 percent of respondents $(n=87)$ reported scores higher than 50, which represents positive feelings toward Muslims.

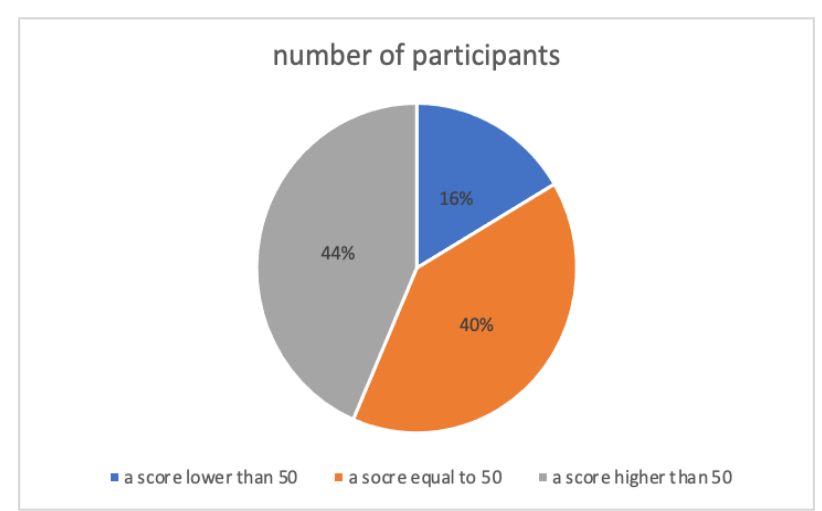

Figure 1 shows the distribution of participant's score of the thermometer. 
In addition, The mean score for the potential threat question was 2.008 , which is below a midpoint of 2.5 on the scale, which indicate that participants of the study have a weak feelings of symbolic threat by Muslims. The mean score for the stereotype question is 2.41 , which is sightly lower than the midpoint of 2.5 , which represents that the participants have a slightly positive stereotypes of muslims as in the study a higher score represent a more negative stereotype. Finally, for questions that are set up to investigate expressed attitude, the mean score is 3.886 . As the expressed attitude questions are measured in a way that a higher score indicates a more positive expressed attitude toward muslims and a score of 2.5 representing neutral attitude. This score represents that in average the participants have a positive expressed attitude toward Muslims.

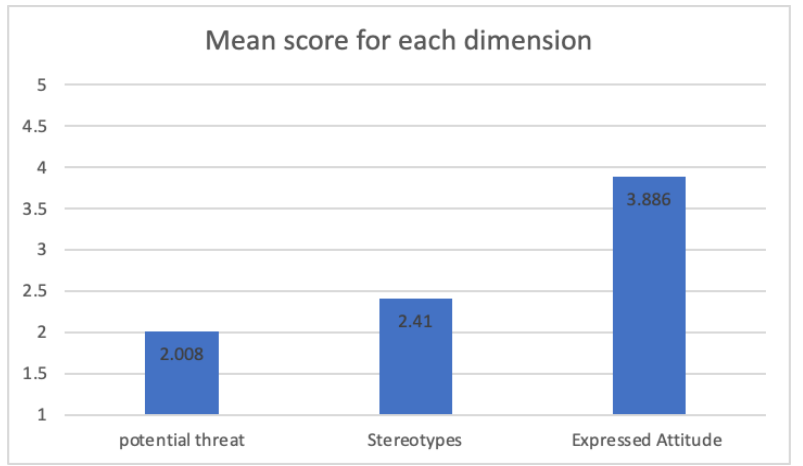

Figure 2 shows the mean score of participants got for potential threat, stereotype and expressed attitude questions

\subsection{Analysis of Factors that Influence Participants' Attitude}

To analyze how the factors like age, gender, religion, ethnic background, and contact with Muslims make differences in participants' attitude toward Muslims, the research team run anova test on SPSS to investigate the statistically significant difference in different groups' attitude.

\subsubsection{Analysis of Age}

Table 1. shows the statistically significant difference between age groups

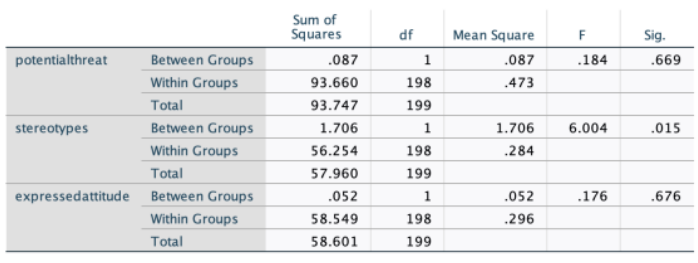

As shown in the table 1, There was a statistically significant difference between age groups in questions about stereotypes at a 0.05 level. More Specifically, as can be seen in figure 3 , the mean score of participants who are $13-17$ is 2.4834 for stereotype questions, which is a higher score than that of respondents who are about 18-22 years old for the same stereotype questions. Thus, as in the study, a higher score indicates more negative stereotypes, the result shows that participants who are younger had a more negative stereotype of Muslims compared to participants who are in the older age group.

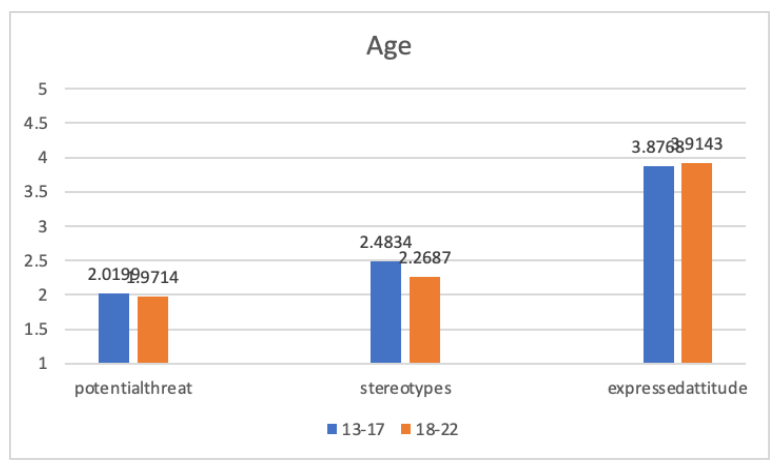

Figure 3 shows the score For Potential Threat, Stereotypes, and The Expressed Attitude Questions of Two Different Age Groups

\subsubsection{Analysis of Sex}

As shown in the table 3, There was no statistically significant sex difference in the questions about potential threat, stereotypes, and expressed attitude at a 0.01 or 0.05 level.

Table 2 shows the sex differences

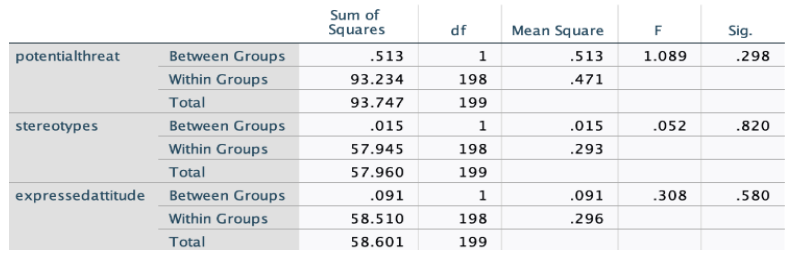

\subsubsection{Analysis of Ethnical Background}

Table 3 shows the difference between ethnical background

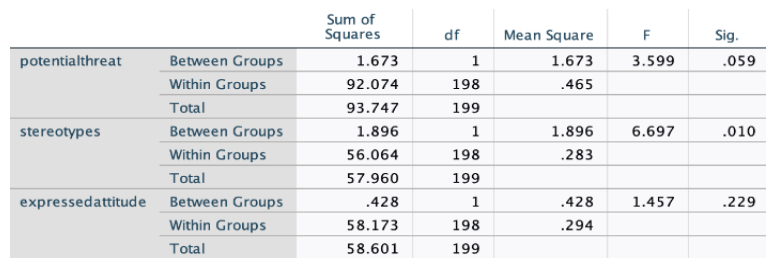

According to the result of table 4, there was a statistically significant difference between different ethnic background, which is divided to 2 category of Han and other ethnical minority groups, in their responses at a 0.01 level. In fact, participants who identify as Han reported an average score of 2.4749 in stereotype questions, which is higher than the score for the same questions reported by participants who identify as one of 
the 55 ethnic minority groups. Because a higher score represents more negative stereotypes toward muslims in the study, it can be concluded from the data that participants who are Han had a more negative stereotype of Muslims compared to the other fifty five ethnic minorities.

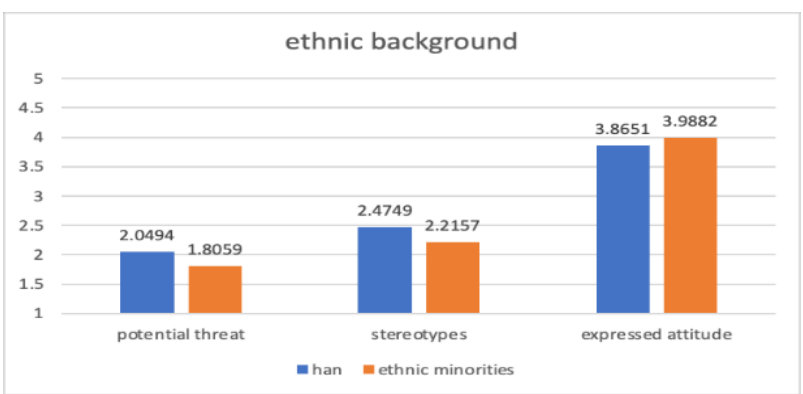

Figure 4. Shows the Score For Potential Threat, Stereotypes, and The Expressed Attitude Questions of Two Different Ethnic Background Groups

\subsubsection{Analysis of Religion}

Table 4 shows differences in the responses of participants with different religion

\begin{tabular}{|c|c|c|c|c|c|c|}
\hline & & $\begin{array}{l}\text { Sum of } \\
\text { Squares }\end{array}$ & df & Mean Square & $F$ & Sig. \\
\hline \multirow[t]{3}{*}{ potentialthreat } & Between Groups & .862 & 2 & .431 & .914 & .402 \\
\hline & Within Groups & 92.885 & 197 & .471 & & \\
\hline & Total & 93.747 & 199 & & & \\
\hline \multirow[t]{3}{*}{ stereotypes } & Between Groups & .679 & 2 & 340 & 1.168 & .313 \\
\hline & Within Groups & 57.281 & 197 & .291 & & \\
\hline & Total & 57.960 & 199 & & & \\
\hline \multirow[t]{3}{*}{ expressedattitude } & Between Groups & 1.266 & 2 & .633 & 2.176 & .116 \\
\hline & Within Groups & 57.334 & 197 & 291 & & \\
\hline & Total & 58.601 & 199 & & & \\
\hline
\end{tabular}

As shown in the table 4, There was no statistically significant difference in how respondents with different degree of religious beliefs in answering the questions about potential threat, stereotypes, and expressed attitude at a 0.01 or 0.05 level.

\subsubsection{Analysis of Intergroup Contact}

Table 5 shows differences in the responses of participants with different level of intergroup contact with muslims

\begin{tabular}{|c|c|c|c|c|c|c|}
\hline & & $\begin{array}{l}\text { Sum of } \\
\text { Squares }\end{array}$ & df & Mean Square & $\mathrm{F}$ & Sig. \\
\hline \multirow[t]{3}{*}{ potentialthreat } & Between Groups & 2.068 & 1 & 2.068 & 4.467 & .036 \\
\hline & Within Groups & 91.679 & 198 & .463 & & \\
\hline & Total & 93.747 & 199 & & & \\
\hline \multirow[t]{3}{*}{ stereotypes } & Between Croups & 3.810 & 1 & 3.810 & 13.932 & $<.001$ \\
\hline & Within Groups & 54.150 & 198 & .273 & & \\
\hline & Total & 57.960 & 199 & & & \\
\hline \multirow[t]{3}{*}{ expressedattitude } & Between Groups & 1.187 & 1 & 1.187 & 4.093 & .044 \\
\hline & Within Groups & 57.414 & 198 & .290 & & \\
\hline & Total & 58.601 & 199 & & & \\
\hline
\end{tabular}

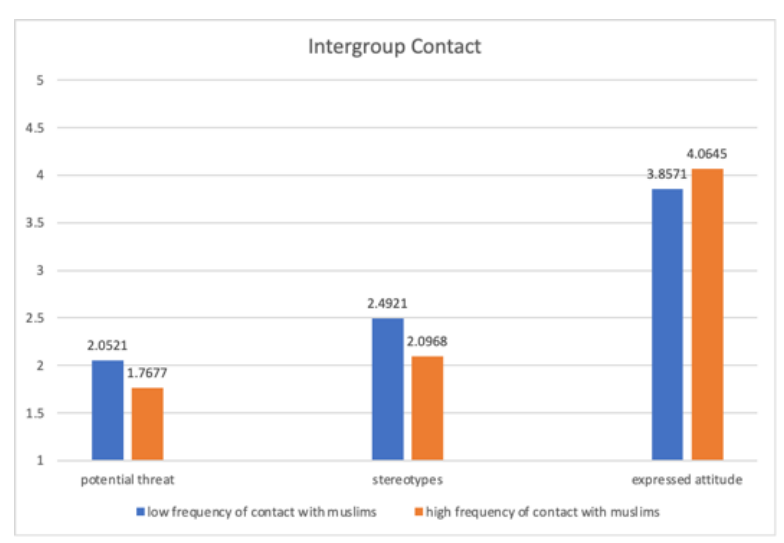

Figure 5 shows Comparison Between Scores For Potential Threat, Stereotypes, and The Expressed Attitude Questions Answered By Group with Low Frequency of Contact with Muslims and The Group with High Frequency of Contact with Muslims.

The result of the analysis shows that there were statistically significant differences between groups who have frequent contact with muslims and no frequent contact in their answers for questions of potential threat, stereotypes, and expressed attitude. From figure 4higher scores $=$ stronger feelings of symbolic threat Participants who have less contact with muslims have stronger feelings of symbolic threat by muslims.

For questions of potential threat, the participants with a low frequency of contact with muslims have a higher average score of 2.0521 than that of respondents with a high frequency of contact with muslims. In the study, a higher scores indicates stronger feelings of symbolic threat [2]. Thus, it is safe to conclude that participants who have less contact with muslims have stronger feelings of symbolic threat by muslims.

For questions of stereotypes toward muslims, participants with low frequency of contact with muslims reported a much higher average score of 2.4921) than participants with a high frequency of contact with muslims, who reported an average score of 2.0968. As for stereotype questions, a higher score indicates a more negative stereotypes. participants who have more contact with muslims had a less negative stereotype of Muslims.

Finally, for questions of expressed attitude, the average score participants with low frequency of contact with muslims reported is a lower than participants with a high frequency of contact with muslims. To be more specific, participants with low frequency of contact with muslims reported a score of 3.8571 while the participants with a high frequency of contact with muslims reported a score of 4.0645 . In the study, a higher score indicates a more positive expressed attitude, so participants who have more contact with muslims had more positive expressed attitude to muslims. This result echo with the previous result of stereotype questions. As participants who have more contact with muslims had a less negative 
stereotype of Muslims, it is natural and reasonable that they would report a more positive expressed attitude toward muslims.

\subsection{Analysis of Factors that Influence Participants' Perception of Others' Attitude Toward Muslims}

Table 6. Age

Table 6. Shows statistically significant difference in how respondents with different age in answering the questions about potential threat, stereotypes, and expressed attitude

\begin{tabular}{|c|c|c|c|c|c|c|}
\hline & & $\begin{array}{l}\text { Sum of } \\
\text { Squares }\end{array}$ & df & Mean Square & $\mathrm{F}$ & Sig. \\
\hline \multirow[t]{3}{*}{ Q27_protriat of muslims } & Between Groups & .828 & 1 & .828 & 1.101 & .295 \\
\hline & Within Groups & 148.927 & 198 & .752 & & \\
\hline & Total & 149.755 & 199 & & & \\
\hline \multirow{3}{*}{$\begin{array}{l}\text { Q28 comments about } \\
\text { muslims }\end{array}$} & Between Croups & 2.535 & 1 & 2.535 & 3.622 & .058 \\
\hline & Within Groups & 138.620 & 198 & .700 & & \\
\hline & Tntal & 141155 & 100 & & & \\
\hline
\end{tabular}

Table 7. Shows statistically significant difference in how respondents with different sex in answering the questions about potential threat, stereotypes, and expressed attitude

\begin{tabular}{|c|c|c|c|c|c|c|}
\hline & & $\begin{array}{l}\text { Sum of } \\
\text { Squares }\end{array}$ & df & Mean Square & $\mathrm{F}$ & Sig. \\
\hline \multirow[t]{3}{*}{ Q27_protriat of muslims } & Between Groups & 2.533 & 1 & 2.533 & 3.407 & .066 \\
\hline & Within Groups & 147.222 & 198 & .744 & & \\
\hline & Total & 149.755 & 199 & & & \\
\hline \multirow{3}{*}{$\begin{array}{l}\text { Q28_comments about } \\
\text { muslims }\end{array}$} & Between Groups & .677 & 1 & .677 & .954 & .330 \\
\hline & Within Groups & 140.478 & 198 & .709 & & \\
\hline & Total & 141155 & 100 & & & \\
\hline
\end{tabular}

Table 8. Shows statistically significant difference in how respondents with different religions in answering the questions about potential threat, stereotypes, and expressed attitude

\begin{tabular}{|c|c|c|c|c|c|c|}
\hline & & $\begin{array}{l}\text { Sum of } \\
\text { Squares }\end{array}$ & df & Mean Square & $\mathrm{F}$ & Sig. \\
\hline \multirow[t]{3}{*}{ Q27_protriat of muslims } & Between Groups & 1.484 & 1 & 1.484 & 1.981 & .161 \\
\hline & Within Groups & 148.271 & 198 & .749 & & \\
\hline & Total & 149.755 & 199 & & & \\
\hline \multirow{3}{*}{$\begin{array}{l}\text { Q28_comments about } \\
\text { muslims }\end{array}$} & Between Groups & 2.462 & 1 & 2.462 & 3.514 & .062 \\
\hline & Within Croups & 138.693 & 198 & .700 & & \\
\hline & Total & 141.155 & 199 & & & \\
\hline
\end{tabular}

As shown in the table 6,7 , and 8 , There was no statistically significant difference in how respondents with different age, sex, and degree of religious beliefs in answering the questions about potential threat, stereotypes, and expressed attitude at a 0.01 or 0.05 level.

However, As shown in the table 9 and 10 respectively, There was statistically significant difference in how respondents with different ethnical backgrounds and extent of intergroup contact with muslims in answering the questions about potential threat, stereotypes, and expressed attitude at a 0.05 or 0.01 level respectively.
Table 9. Shows statistically significant difference in how respondents with different ethnic backgrounds in answering the questions about potential threat, stereotypes, and expressed attitude

\begin{tabular}{|c|c|c|c|c|c|c|}
\hline & & $\begin{array}{l}\text { Sum of } \\
\text { Squares }\end{array}$ & df & Mean Square & $\mathrm{F}$ & Sig. \\
\hline \multirow[t]{3}{*}{ Q27_protriat of muslims } & Between Croups & 4.049 & 1 & 4.049 & 5.503 & .020 \\
\hline & Within Croups & 145.706 & 198 & .736 & & \\
\hline & Total & 149.755 & 199 & & & \\
\hline \multirow{3}{*}{$\begin{array}{l}\text { Q28_comments about } \\
\text { muslims }\end{array}$} & Between Croups & .813 & 1 & .813 & 1.147 & .285 \\
\hline & Within Groups & 140.342 & 198 & .709 & & \\
\hline & Total & 141.155 & 199 & & & \\
\hline
\end{tabular}

Table 10. Shows statistically significant difference in how respondents with different level of intergroup contact with muslims in answering the questions about potential threat, stereotypes, and expressed attitude

\begin{tabular}{|c|c|c|c|c|c|c|}
\hline & & $\begin{array}{l}\text { Sum of } \\
\text { Squares }\end{array}$ & df & Mean Square & $\mathrm{F}$ & Sig. \\
\hline \multirow[t]{3}{*}{ Q27_protriat of muslims } & Between Croups & 6.788 & 1 & 6.788 & 9.402 & .002 \\
\hline & Within Croups & 142.967 & 198 & .722 & & \\
\hline & Total & 149.755 & 199 & & & \\
\hline \multirow{3}{*}{$\begin{array}{l}\text { Q28 comments about } \\
\text { muslims }\end{array}$} & Between Croups & .768 & 1 & .768 & 1.083 & .299 \\
\hline & Within Groups & 140.387 & 198 & .709 & & \\
\hline & Total & 141.155 & 199 & & & \\
\hline
\end{tabular}

Specifically, participants who identify as Han people gave an average score of 2.15 , which is lower than the average score of 2.53, which was reported by the respondents who identify as one of the 55 ethnic minority groups in China. As a higher score represents a perception of a more positive portrait of muslims in major social medias in China, it is reasonable to conclude that Han people perceive more negative portrait of muslims from major social medias in China.

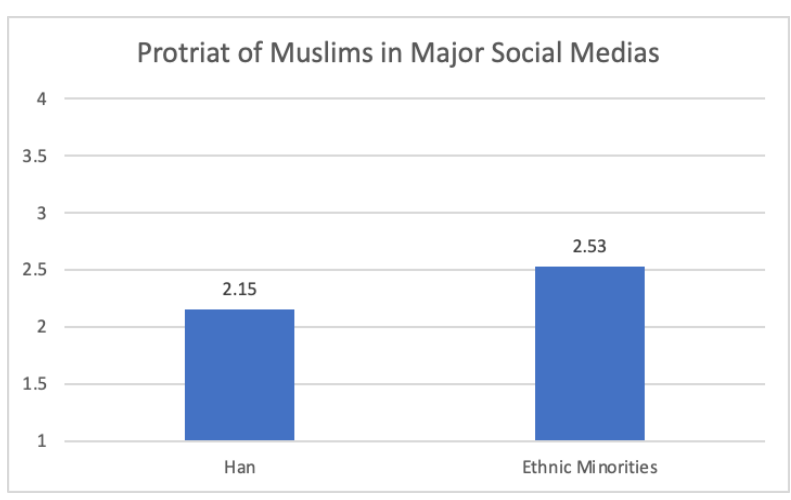

Figure 6 shows Comparison Between Average Scores For Question 27 Answered By Han and Other Ethnic Minorities in China.

In addition, as shown in figure 6, there was a statistically significant difference between people with low frequency of contact and people with high frequency of contact with muslims in their perception of the portrait of muslims in major social medias in China. Respondents with low frequency of contact with muslims report a lower score of 2.14 compared to the average score of 2.65 reported by participants with a high frequency of contact 
with muslims. As a higher score represents a perception of a more positive portrait of muslims in major social medias in China, respondents with lower frequency of contact with Muslims perceive more negative portrait of muslims from major social medias in China. Again, this result echos with the previous results of the study. As it is concluded that participants who have more contact with muslims had a less negative stereotype of Muslims, it is reasonable that they would be more likely to be exposed to and accept a more positive portrait of muslims in major social medias.

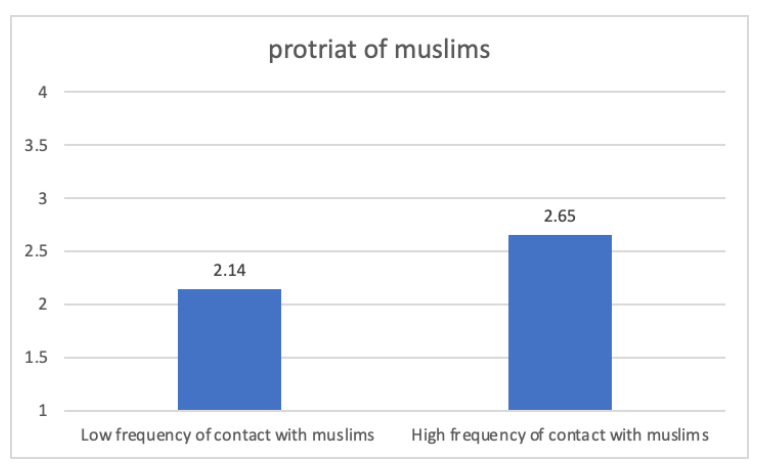

Figure 7 shows Comparison Between Average Scores For Question 27 Answered By Groups with Different Extent of Intergroup Contact with Muslims.

\section{DISCUSSION}

\subsection{General Trends}

Overall, there is an average positive expressed attitude toward Muslims in China in the study. Although there are studies in western countries that show that ingroup identification was found to be positively associated with symbolic threat toward muslims, our results did not align with this such a conclusion.

\subsection{Application of The Study}

In the study, the researchers found that more intergroup contact with Muslims is highly associated with less negative stereotypes and more positive expressed attitude toward muslims. This result is consistent with other research by Pettigrew and Tropp, which shows that contact leads to decategorization, increasing knowledge about the out-group, and thereby to less stereotyping [3]. Thus, such a finding may inspire educators and government officials to come up potential solutions to eliminate stereotypes and negative feelings against Chinese Muslims by creating more intergroup contact. For example, courses about Islamic culture and Muslims' lives in China can be taught from elementary schools to universities. In addition, government should revise or come up solutions that promote a greater ethnic diversity in workplaces and schools.

\subsection{Evaluation}

There are several limitations within the research that decreases the validity and reliability of the results being collected and therefore the conclusions that arrived upon.

First of all, the research can only collect and analyze participants' self reported expressed attitude toward muslims, which can be biased as the implicit bias and prejudice can not be measured through the study. In addition, the research sample is quite restricted with mostly Han people. To improve the study, a bigger sample of more ethic minorities groups should be needed, as the differences in ethnical background make great differences in people's attitude.

As the study relied on online questionnaire, there can be volunteer bias, which can decrease the validity of the results. For example, people who have a more positive attitude toward muslims may be more likely to answer the questionnaire as they have no fear of being judged. Thus, the sample can be biased due to the design.

\section{CONCLUSION}

Despite the limitation of the study, there are some worth mentioning results. For example, research team can conclude that there is a generally positive expressed attitude toward muslims in the younger generation. The researchers also found that more intergroup contact with Muslims is highly associated with less negative stereotypes and more positive expressed attitude toward muslims, which echo with other researchers' work and provide insights into how to eliminate stereotypes and improve the intergroup relationship between Chinese Muslims and others.

\section{REFERENCES}

[1] Luwei Rose Luqiu, Fan Yang (2018). Islamophobia in China: news coverage, stereotypes, and Chinese Muslims' perceptions of themselves and Islam, Asian Journal of Communication, 28:6, 598-619, DOI: $\underline{10.1080 / 01292986.2018 .1457063}$

[2] Velasco Gonzalez, Karina, et al. (2008). Prejudice towards Muslims in The Netherlands: Testing Integrated Threat Theory. British Journal of Social Psychology, vol. 47, no. 4, 667-685, DOI: $10.1348 / 014466608 \times 284443$.

[3] Pettigrew, T., \& Tropp, L. (2006). A meta-analytic test of intergroup contact theory. Journal of Personality and Social Psychology, 90, 751-783. 